\title{
Quality of life in women with breast cancer, after surgical intervention, in a city in the zona da mata region in Minas Gerais, Brazil
}

Priscila Almeida Barbosa 1

Rhodrigo Goldner Cesca 2

Thatiana Egizi Dias Pacífico 3

Isabel Cristina Gonçalves Leite 4

1-4 Universidade Federal de Juiz de Fora. Rua José Lourenço Kelmer, s.n. Martelos. Juiz de Fora, MG, Brasil. CEP: 36.036-330.

\begin{abstract}
Objectives: To evaluate health-related quality of life (HRQoL) in women with breast cancer, after surgical intervention, attended at the regional Oncology Referral Hospital in Juiz de Fora (MG).

Methods: 121 women were evaluated and the European Organization for Research and Treatment of Cancer, Breast Cancer-Specific Quality of Life Questionnaire (EORTC QLC 30 BR23) was applied. After the descriptive analysis, a multiple linear regression model was created. The SPSS 14 was used to build the database and to conduct the analysis.

Results: the mean values of the EORTC QLC 30 BR23 showed a tendency for good HRQoL, thus, the functional scale showed the most impairment with the worst mean scores observed. There was statistical significance among the symptoms scale and obese women who underwent adjuvant radiotherapy and those who had a greater number of dissected lymph nodes. The subjective perceptions influenced all the EORTC QLC 30 BR23domains.

Conclusions: after controlling the variable by functional hand, and time of surgery, the BMI variables, breast reconstruction, heavy feeling in the arm, range of motion (ROM) reduction of the shoulder, and the tight shirt-sleeve sensation proven capable of explaining HRQoL. HRQoL appears to be relatively good, but negatively influenced by subjective perceptions, in addition to treatment factors.

Key words Women's health, Breast neoplasms, Quality of life, Lymphedema
\end{abstract}




\section{Introduction}

World widely, breast cancer is the most frequent tumor in women, accounting for $25 \%$ of the neoplasms of this population group in 2012. In Brazil, around 57,960 women will be diagnosed with breast cancer in 2016, of which 29,760 will be in the Southeast alone. 1 As one of the main causes of morbimortality with great epidemiological importance and relevant social impact, becoming an important focus for health care. ${ }^{2}$

In Brazil, failures in screening strategies and difficulties to access treatment lead to late diagnosis in advanced tumor stages, ${ }^{2-3}$ resulting in a more aggressive surgical approach as the most recommended therapeutic alternative and, consequently, an increase in the physical, psychological, and social sequelae. ${ }^{4}$

The deterioration that the diagnosis and treatment of breast cancer brings to a woman's quality of life $(\mathrm{QoL})$ has been evident in many studies in recent years, not only in the context of morbidities, but also in relation to psychosocial aspects such as the fear of recurrence or even death, difficulties in carrying on daily life activities, changes in body image, sexual dysfunction, and changes in thelifestyle. 4

The technological advances for breast cancer in the diagnosis and treatment have increased the survival of patients with cancer, that is, adding years to one's life, but this does not add life to the years, ${ }^{4}$ thus placing value on a relevant research aspect in recent times: The quality of life of patients with breast neoplasia after surgical intervention. ${ }^{5}$

Although there is no consensus regarding the definition of the quality of life concept, given the complexity of variables related to education, finances, and sociocultural factors involved, the authors agree that these three aspects should be addressed: subjectivity, multidimensionality, and bipolarity. ${ }^{4}$ In the health area, therefore, there is a tendency to recognize the importance of the patient's point of view in relation to the impact of the illness, the impairment, and the therapeutic interventions, in the quality of life context, that is, health-related quality of life (HRQoL).

Currently, there is a wide variety of generic and specific instruments for assessing quality of life. The generic instruments are used for overall assessment of important aspects related to QoL (physical, social, psychological, spiritual) in any health condition, while the specific ones are more sensitive in detecting changes after an intervention, allowing to assess a specific condition of a determined function, population, or disease. The use of both, generic and specific, is recommended in order to combine advantages and minimize the disadvantages of each instrument. ${ }^{5}$ Among the numerous of specific instruments available for evaluating quality of life related to breast cancer, the EORTC QLC 30 BR23 stands out as the most commonly used in the literature, ${ }^{6}$ as it has presented proven reproducibility and reliability. ${ }^{7}$

The city of Juiz de Fora is a reference in the health area, mainly in oncology, although the quality of life of women with breast cancer is still unknown in this city. Therefore, considering the importance of the topic in the health area, this study intends to evaluate women's quality of life of a after surgical intervention for breast cancer, in order to identify the potential variables which influence them. The expectation is that the results obtained will help increase scientific knowledge, enabling the development of therapies that promote better adaptation to the disease and to the oncological treatment, and the actions that minimize the complications arising from the breast neoplasm treatment, whether they are physical, social, or psychological.

\section{Methods}

This is a cross-sectional, analytical study intended to assess the quality of life of women with breast cancer, after surgical intervention, treated at the regional Oncology Referral Hospital in Juiz de Fora, Minas Gerais. The information was collected between January and October 2013.

The study included women in breast cancer treatment after more than six months since surgical intervention, who appeared at the Mastology and Oncology Outpatient Clinic for follow-up consultation during the period and at the defined location for the study. Women with bilateral breast cancer, localregional or distant active disease, functional alteration of the upper limbs before the cancer treatment, and the absence of axillary intervention (partial or total axillary lymphadenectomy and/or sentinel lymph node biopsy) were excluded.

After the calculation, according to EpiInfo, 3.5.2 ${ }^{\circledR}$, a minimum of 111 cases was estimated in order to constitute the study. During the data collecting phase, 134 women were approached and invited to participate in this study; 8 did not meet the inclusion criteria on the interview date; 5 women refused to participate; thus a total of 121 women constituted the final sample.

The recruited cases were carried out by analyzing clinical records of patients who began breast cancer treatment between January 2007 and December 2012, at the study location. By analyzing 
the medical records, tumor-related and treatmentrelated variables were evaluated.

After identifying the patients meeting the inclusion criteria, they were informed as to the type of study and how it would be conducted, through the Informed Consent Form. The study was previously approved by the Human Research Ethics Committee at the Federal University of Juiz de Fora, under the document number 135.887 .

The women who agreed to participate in the study were interviewed by the researcher in charge, which consisted primarily of a patient profile chart: identification, sociodemographic data, and surgical complications. Next, the QoL questionnaire of the European Organization for Research and Treatment of Cancer, Breast Cancer-Specific Quality of Life Questionnaire (EORTC QLC 30 BR-23) was applied.7

The EORTC - C30, a generic part of the QoL questionnaire, is multidimensional and aims to assess the QoL in cancer patients during the previous two weeks. The first part is composed of 30 items that address general questions about cancer, such as: symptoms, side effects of the treatment, psychological distress, physical functioning, social interaction, sexuality, body image, overall health, quality of life, and satisfaction with medical care, regardless to the type of cancer.

The specific EORTC - BR 23 module aims to evaluate patients treated for breast cancer or undergoing treatment. This should be used together with the EORTC - C30. The EORTC-BR 23 scale has 23 questions, with continuity from the general questionnaire, and incorporates the following two scales: the functional scale, which covers body image and sexual function; and the symptoms scale, which focuses on the symptoms of the arm and breast, and the treatment effects.

Finally, a physical evaluation questionnaire was applied, which included the analysis of the presence of lymphedema, measured according to the CasleySmith proposal, ${ }^{8}$ with the estimated limb volume being calculated from the five circumference measurements, treating each segment of the limb as a pair of circumferences (truncated cone).

The study information was submitted to descriptive analysis to obtain measurements of the central tendency (mean) and of the dispersion (standard deviation) for the continuous variables, and the absolute and relative frequencies for the nominal variables. The calculations of the mean scores for each EORTC QLC30 BR23 scale followed the instructions described in the questionnaire manual.

After the description, the univariate analysis was performed to test the association between each of the independent variables and the dependent variable, QoL. For those that had a normal distribution, the Student's t-test was used; for the nominal variables, the chi-square $\left(\chi^{2}\right)$ was used; and the Mann-Whitney U test and Kruskal Wallis test for quantitative variables with non-parametric distribution.

In the multivariate analysis, multiple linear regression models were constructed for the analysis of the independent variables associated with the outcome. The variables with $p<0.20$ from the univariate analysis, as well as those with clinical relevance, controlled for confounding factors (functional hand and time since surgery), were admitted. The final model admitted variables with $p<0.05$. Finally, the Statistical Package for Social Sciences (SPSS), version 14, was used for the statistical analysis of the information.

\section{Results}

Regarding the sociodemographic characterization of the evaluated sample, it was verified that $71.1 \%$ of the women were over 50 years of age, with a mean of 56.99 years $(\mathrm{SD}= \pm 10.45)$. The majority were white $(76 \%)$, not married $(52.9 \%)$, and had completed at least elementary school (54.5\%). Regarding to occupation, as of the interview date, $71.9 \%$ reported being homemakers or retired; analyzing their occupation before treatment, $73.6 \%$ were included in the labor market (Table 1).

As for the clinical variable BMI, approximately $60.3 \%$ of the study population was overweight, with a mean BMI of $27.36 \mathrm{~kg} / \mathrm{cm}^{2}(\mathrm{SD}= \pm 5.20)$ (Table 1).

In relation to the tumor and treatment variables, conservative breast surgery was the most common $(65.3 \%)$, most of the women were diagnosed in advanced stages III and IV (76\%) and had an average of less than 12 lymph nodes removed $(50.4 \%)$. It was observed that $14.9 \%$ had done breast reconstruction and, of these, $88.2 \%$ were immediate. The frequency of adjuvant treatment was high in the sample, with $81 \%$ underwent chemotherapy, $84.3 \%$ underwent radiotherapy, and $86.8 \%$ underwent hormone therapy. About $62 \%$ of the women presented time elapsed between the surgery and the interview date of over 2 years, with an average of 2.84 years $(\mathrm{SD}= \pm 1.54)$. Regarding the physical evaluation of the 121 women evaluated, 9 (7.4\%) presented lymphedema in the upper limb homolateral to surgical intervention of the breast (Table 1).

The mean values found for each module of the EORTC 30 and BR 23 questionnaires, a high mean 
Description of the study sample according to the variables listed, in women with breast cancer, after surgical treatment. Juiz de Fora, Minas Gerais, Brazil, 2013.

\begin{tabular}{|c|c|c|}
\hline \multirow{2}{*}{ Variables } & \multicolumn{2}{|c|}{$($ Total = 121) } \\
\hline & $\mathrm{n}$ & $\%$ \\
\hline \multicolumn{3}{|l|}{ Age (years) } \\
\hline Up to 50 & 35 & 28.9 \\
\hline$>50$ & 86 & 71.1 \\
\hline \multicolumn{3}{|l|}{ Marital status } \\
\hline Married & 57 & 47.1 \\
\hline Not married & 64 & 52.9 \\
\hline \multicolumn{3}{|l|}{ Education level } \\
\hline Up to elementary & 66 & 54.5 \\
\hline Above elementary & 55 & 45.5 \\
\hline \multicolumn{3}{|l|}{ Skin color } \\
\hline White & 92 & 76.0 \\
\hline Non-white & 29 & 24.0 \\
\hline \multicolumn{3}{|l|}{ Current occupation } \\
\hline Actively employed & 34 & 28.1 \\
\hline Homemaker / retired & 87 & 71.9 \\
\hline \multicolumn{3}{|c|}{ Occupation before treatment } \\
\hline Actively employed & 89 & 73.6 \\
\hline Homemaker / retired & 32 & 26.4 \\
\hline \multicolumn{3}{|l|}{ BMI } \\
\hline Eutrophic $(<25)$ & 42 & 34.7 \\
\hline Overweight (25 - 30) & 43 & 35.5 \\
\hline Obese $(>30)$ & 30 & 24.8 \\
\hline No information & 6 & 5.0 \\
\hline \multicolumn{3}{|l|}{ Clinical stage } \\
\hline $0-I I$ & 29 & 24.0 \\
\hline III-IV & 92 & 76.0 \\
\hline \multicolumn{3}{|l|}{ Type of surgery } \\
\hline Conservative & 79 & 65.3 \\
\hline Non-conservative & 38 & 31.4 \\
\hline No information & 4 & 3.3 \\
\hline \multicolumn{3}{|c|}{ Type of axillary lymphadenectomy performed } \\
\hline SLB & 34 & 28.1 \\
\hline PAD & 38 & 31.4 \\
\hline RAD & 38 & 31.4 \\
\hline No information & 11 & 9.1 \\
\hline \multicolumn{3}{|l|}{ Lymph nodes removed } \\
\hline less than 12 (mean) & 61 & 50.4 \\
\hline 12 or more & 49 & 40.5 \\
\hline No information & 11 & 9.1 \\
\hline \multicolumn{3}{|l|}{ Breast Reconstruction } \\
\hline Yes & 18 & 14.9 \\
\hline No & 103 & 85.1 \\
\hline
\end{tabular}

SLB = sentinel lymph node biopsy; $P A D=$ partial axillary dissection; $R A D=$ radical axillary dissection; $B M I=$ body mass index.

Notes: Married: with partner or in a stable union; Not married: divorced or separated, widowed or unmarried; Up to elementary: has a complete elementary education or less; Above elementary: has a high school or college education level. 
Description of the study sample according to the variables listed, in women with breast cancer, after surgical treatment. Juiz de Fora, Minas Gerais, Brazil, 2013.

\begin{tabular}{|c|c|c|}
\hline \multirow{2}{*}{ Variables } & \multicolumn{2}{|c|}{$($ Total $=121)$} \\
\hline & $\mathrm{n}$ & $\%$ \\
\hline \multicolumn{3}{|c|}{ Adjuvant chemotherapy } \\
\hline Yes & 98 & 81.0 \\
\hline No & 22 & 18.2 \\
\hline No information & 1 & 0.8 \\
\hline \multicolumn{3}{|l|}{ Adjuvant radiotherapy } \\
\hline Yes & 102 & 84.3 \\
\hline No & 18 & 14.9 \\
\hline No information & 1 & 0.8 \\
\hline \multicolumn{3}{|l|}{ Hormone Therapy } \\
\hline Yes & 105 & 86.8 \\
\hline No & 15 & 12.4 \\
\hline No information & 1 & 0.8 \\
\hline \multicolumn{3}{|l|}{ Time since Surgery } \\
\hline 2 years or less & 43 & 35.5 \\
\hline more than 2 years & 75 & 62.0 \\
\hline No information & 3 & 2.5 \\
\hline \multicolumn{3}{|l|}{ Lymphedema } \\
\hline Present & 9 & 7.4 \\
\hline Absent & 112 & 92.6 \\
\hline
\end{tabular}

SLB = sentinel lymph node biopsy; PAD = partial axillary dissection; RAD = radical axillary dissection; $B M I=$ body mass index.

Notes: Married: with partner or in a stable union; Not married: divorced or separated, widowed or unmarried; Up to elementary: has a complete elementary education or less; Above elementary: has a high school or college education level.

score of $75.2(\mathrm{SD}= \pm 22.11)$ was found on the overall health scale of the generic EORTC 30 module, indicating a tendency towards a good quality of life. The functional scale of the EORTC 30 was the most compromised, with a lower mean score of 28.2 (SD $= \pm 21.02)$. In the specific module for breast cancer (BR23), the symptom scale obtained a low mean score, 25.7 ( $\mathrm{SD}= \pm 21.05)$, indicating a lower amount of symptoms and a better quality of life.

No statistical significance was observed in the analysis of the association between the mean scores of the EORTC QLC30 BR23 and the sociodemographic variables (Table 2).

Upon comparing the other clinical variables, tumor, and treatment with the mean scores of the EORTC QLC 30 and BR 23 scales (Table 3), found that the clinical variable BMI influenced both symptom scales of the instrument, with the highest mean scores (24.8 and 36.7, respectively, with $p=0.029$ and $p=0.015$ ) observed among obese individuals, indicating a negative influence of obesity on quality of life. Radiotherapy showed a significant association with the EORTC QLC 30 symptom scale, indicating a poorer quality of life among those who underwent radiotherapy $(p=0.052)$. In addition, tangential value was observed between radiotherapy and the BR23 functional scale, with a better quality of life observed among those who did not undergo radiotherapy $(p=0.06)$. In relation to the number of dissected lymph nodes, there was a significant association with the symptom scale of the specific BR23 module, denoting poorer quality of life among those with a higher number of lymph nodes removed $(p=0.024)$. In this study, there were no significant associations regarding the type of surgery, clinical stage, axillary lymphadenectomy, chemotherapy, hormone therapy, and time since surgery in the EORTC QLC 30 and BR 23 modules (Table 3).

When evaluating the mean scores of the EORTC QLC 30 and BR 23 modules regarding subjective perceptions for the diagnosis of lymphedema in relation to the time elapsed (Table 4), a high significance was noted in most of the scales $(p<0.001)$, indicating an influence of the subjective diagnosis of 
Table 2

The relation of the means from the EORTC QLC 30 BR 23 questionnaire scales with the sociodemographic variables, in women with breast cancer, after surgical treatment. Juiz de Fora, Minas Gerais, Brazil, 2013.

\begin{tabular}{|c|c|c|c|c|c|c|c|c|c|c|c|c|c|c|c|}
\hline \multirow{3}{*}{ Variables } & \multirow{2}{*}{\multicolumn{3}{|c|}{ Overall Health }} & \multicolumn{6}{|c|}{ EORTC 30} & \multicolumn{6}{|c|}{ EORTC BR23 } \\
\hline & & & & \multicolumn{3}{|c|}{ Functional } & \multicolumn{3}{|c|}{ Symptom } & \multicolumn{3}{|c|}{ Functional } & \multicolumn{3}{|c|}{ Symptom } \\
\hline & M & SD & $p$ & M & SD & $p$ & $M$ & SD & $p$ & M & SD & $p$ & M & SD & $p$ \\
\hline Age (years) & & & 0.444 & & & 1.000 & & & 0.667 & & & 0.889 & & & 0.444 \\
\hline$\leq 50$ & 66.7 & - & & 48.9 & - & & 35.9 & - & & 62.5 & - & & 69.0 & - & \\
\hline$>50$ & 79.2 & \pm 16.7 & & 43.6 & \pm 24.7 & & 25.0 & \pm 15.9 & & 58.3 & \pm 28.7 & & 39.6 & \pm 19.8 & \\
\hline Marital status & & & 1.000 & & & 0.556 & & & 0.556 & & & 0.556 & & & 1.000 \\
\hline Married & 76.7 & \pm 18.0 & & 40.0 & \pm 27.3 & & 23.6 & \pm 19.1 & & 60.9 & \pm 27.5 & & 40.0 & \pm 21.0 & \\
\hline Not married & 79.2 & \pm 15.9 & & 49.4 & \pm 19.2 & & 29.5 & \pm 10.4 & & 56.1 & \pm 29.5 & & 46.4 & \pm 23.4 & \\
\hline Education level & & & 0.190 & & & 0.556 & & & 0.730 & & & 0.730 & & & 0.063 \\
\hline Up to elementary & 70.0 & \pm 12.6 & & 48.4 & \pm 20.9 & & 28.7 & \pm 17.3 & & 55.2 & \pm 19.4 & & 55.2 & \pm 19.4 & \\
\hline Above elementary & 87.5 & \pm 15.9 & & 23.0 & \pm 34.4 & & 23.0 & \pm 14.0 & & 66.7 & \pm 9.8 & & 27.4 & \pm 9.8 & \\
\hline Skin color & & & 0.500 & & & 0.889 & & & 0.889 & & & 0.667 & & & 0.500 \\
\hline White & 75.0 & \pm 15.9 & & 44.1 & \pm 26.3 & & 24.9 & \pm 17.3 & & 59.3 & \pm 27.8 & & 45.9 & \pm 22.9 & \\
\hline Non-white & 87.5 & \pm 17.8 & & 44.4 & \pm 12.6 & & 30.8 & \pm 3.6 & & 57.2 & \pm 33.7 & & 32.1 & \pm 8.4 & \\
\hline Current occupation & & & 0.889 & & & 0.889 & & & 0.500 & & & 0.333 & & & 0.222 \\
\hline Homemaker / retired & 75.0 & \pm 35.3 & & 47.8 & \pm 45.6 & & 35.9 & \pm 21.7 & & 71.4 & \pm 40.4 & & 57.1 & \pm 23.6 & \\
\hline Actively employed & 78.6 & \pm 11.6 & & 43.2 & \pm 19.1 & & 23.4 & \pm 13.8 & & 55.2 & \pm 24.9 & & 38.8 & \pm 20.1 & \\
\hline Occupation before treatment & & & 1.000 & & & 0.556 & & & 0.556 & & & 0.556 & & & 1.000 \\
\hline Homemaker / retired & 76.7 & \pm 18.0 & & 40.0 & \pm 27.3 & & 23.6 & \pm 19.1 & & 60.9 & \pm 27.8 & & 40.0 & \pm 21.0 & \\
\hline Actively employed & 79.2 & \pm 16.0 & & 49.4 & \pm 19.2 & & 29.5 & \pm 10.4 & & 56.1 & \pm 29.5 & & 46.4 & \pm 23.4 & \\
\hline
\end{tabular}

$M=$ mean; $S D=$ standard deviation; $p=$ significance probability .

EORTC QLC = European Organization for Research and Treatment of Cancer, Breast Cancer-Specific Quality of Life Questionnaire. 
The relation of the means from the EORTC QLC 30 BR 23 questionnaire scales with the clinical, tumor and treatment variables, in women with breast cancer, after surgical treatment. Juiz de Fora, Minas Gerais, Brazil, 2013.

\begin{tabular}{|c|c|c|c|c|c|c|c|c|c|c|c|c|c|c|c|}
\hline \multirow{3}{*}{ Variables } & & & & \multicolumn{6}{|c|}{ EORTC 30} & \multicolumn{6}{|c|}{ EORTC BR23 } \\
\hline & \multicolumn{3}{|c|}{ Overall Health } & \multicolumn{3}{|c|}{ Functional } & \multicolumn{3}{|c|}{ Symptom } & \multicolumn{3}{|c|}{ Functional } & \multicolumn{3}{|c|}{ Symptom } \\
\hline & M & SD & $p$ & M & SD & $p$ & M & SD & $p$ & M & SD & $p$ & M & SD & $p$ \\
\hline BMI & & & 0.265 & & & 0.189 & & & 0.029 & & & 0.501 & & & 0.015 \\
\hline Eutrophic & 79.2 & \pm 20.1 & & 23.6 & \pm 16.8 & & 15.8 & \pm 16.6 & & 41.4 & \pm 18.3 & & 21.1 & \pm 19.6 & \\
\hline Overweight & 71.5 & \pm 24.2 & & 28.7 & \pm 20.9 & & 23.4 & \pm 16.2 & & 46.5 & \pm 22.8 & & 23.2 & \pm 16.4 & \\
\hline Obese & 73.0 & \pm 20.9 & & 35.6 & \pm 25.5 & & 24.8 & \pm 19.9 & & 48.8 & \pm 25.6 & & 36.7 & \pm 26.2 & \\
\hline Conservative surgery & & & 0.901 & & & 0.746 & & & 0.645 & & & 0.206 & & & 0.324 \\
\hline Yes & 75.0 & \pm 22.6 & & 28.9 & \pm 21.8 & & 21.5 & \pm 18.8 & & 42.7 & \pm 20.7 & & 24.4 & \pm 20.4 & \\
\hline No & 75.6 & \pm 21.4 & & 26.8 & \pm 19.7 & & 19.1 & \pm 15.8 & & 48.7 & \pm 23.6 & & 28.1 & \pm 22.2 & \\
\hline Stage & & & 0.113 & & & 0.116 & & & 0.256 & & & 0.925 & & & 0.095 \\
\hline $0-11$ & 76.4 & \pm 23.7 & & 27.1 & \pm 22.1 & & 22.5 & \pm 19.5 & & 40.9 & \pm 18.0 & & 21.3 & \pm 19.1 & \\
\hline III-IV & 74.8 & \pm 21.7 & & 28.5 & \pm 20.8 & & 20.1 & \pm 17.3 & & 46.0 & \pm 22.9 & & 27.1 & \pm 21.5 & \\
\hline Axillary lymphadenectomy & & & 0.420 & & & 0.102 & & & 0.360 & & & 0.468 & & & 0.210 \\
\hline SLB & 82.1 & \pm 22.5 & & 22.0 & \pm 19.9 & & 17.3 & \pm 17.5 & & 40.3 & \pm 19.7 & & 18.1 & \pm 16.7 & \\
\hline PAD & 69.5 & \pm 25.8 & & 32.2 & \pm 22.4 & & 23.6 & \pm 19.9 & & 46.1 & \pm 23.4 & & 32.2 & \pm 26.1 & \\
\hline RAD & 74.3 & \pm 17.5 & & 28.7 & \pm 20.1 & & 20.9 & \pm 16.1 & & 47.0 & \pm 21.8 & & 26.6 & \pm 17.7 & \\
\hline Lymph nodes removed - less than & & & 0.149 & & & 0.645 & & & 0.405 & & & 0.149 & & & 0.024 \\
\hline 12 (mean) & & & & & & & 20.5 & \pm 20.4 & & 42.8 & \pm 21.6 & & 23.5 & \pm 23.3 & \\
\hline Yes & 76.2 & \pm 25.0 & & 28.0 & \pm 23.3 & & 21.0 & \pm 14.5 & & 49.1 & \pm 22.6 & & 29.8 & \pm 19.5 & \\
\hline No & 72.6 & \pm 18.6 & & 28.5 & \pm 18.9 & & & & & & & & & & \\
\hline Breast Reconstruction & & & 0.312 & & & 0.175 & & & 0.083 & & & 0.167 & & & 0.762 \\
\hline Yes & 69.4 & \pm 24.6 & & 22.0 & \pm 19.6 & & 15.7 & \pm 18.9 & & 40.1 & \pm 22.6 & & 27.7 & \pm 24.3 & \\
\hline No & 76.2 & \pm 21.6 & & 29.2 & \pm 21.2 & & 21.5 & \pm 17.5 & & 45.6 & \pm 21.7 & & 25.3 & \pm 20.5 & \\
\hline
\end{tabular}

$M=$ mean; $S D=$ standard deviation; $p=$ significance probability

$\mathrm{SLB}=$ sentinel lymph node biopsy; $\mathrm{PAD}=$ partial axillary dissection; $\mathrm{RAD}=$ radical axillary dissection; $\mathrm{BMI}=$ body mass index

EORTC QLC = European Organization for Research and Treatment of Cancer, Breast Cancer-Specific Quality of Life Questionnaire. 
The relation of the means from the EORTC QLC 30 BR 23 questionnaire scales with the clinical, tumor and treatment variables, in women with breast cancer, after surgical treatment. Juiz de Fora, Minas Gerais, Brazil, 2013.

\begin{tabular}{|c|c|c|c|c|c|c|c|c|c|c|c|c|c|c|c|}
\hline \multirow{3}{*}{ Variables } & & & & \multicolumn{6}{|c|}{ EORTC 30} & \multicolumn{6}{|c|}{ EORTC BR23 } \\
\hline & \multicolumn{3}{|c|}{ Overall Health } & \multicolumn{3}{|c|}{ Functional } & \multicolumn{3}{|c|}{ Symptom } & \multicolumn{3}{|c|}{ Functional } & \multicolumn{3}{|c|}{ Symptom } \\
\hline & M & SD & $p$ & M & SD & $p$ & M & SD & $p$ & M & SD & $p$ & M & SD & $p$ \\
\hline Chemotherapy & & & 0.166 & & & 0.512 & & & 0.919 & & & 0.166 & & & \\
\hline Yes & 74.1 & \pm 21.1 & & 28.5 & \pm 20.5 & & 20.6 & \pm 17.5 & & 46.4 & \pm 22.5 & & 26.6 & \pm 21.1 & 0.208 \\
\hline No & 79.2 & \pm 26.2 & & 26.8 & \pm 24.0 & & 21.1 & \pm 19.8 & & 38.3 & \pm 18.1 & & 21.7 & \pm 21.3 & \\
\hline Radiotherapy & & & 0.424 & & & 0.052 & & & 0.311 & & & 0.060 & & & \\
\hline Yes & 75.7 & \pm 21.7 & & 27.5 & \pm 20.6 & & 19.9 & \pm 17.5 & & 43.3 & \pm 21.4 & & 25.0 & \pm 20.8 & 0.362 \\
\hline No & 70.8 & \pm 24.3 & & 32.0 & \pm 24.2 & & 24.9 & \pm 19.6 & & 54.1 & \pm 23.1 & & 29.8 & \pm 23.1 & \\
\hline Hormone therapy & & & 0.516 & & & 0.409 & & & 0.768 & & & 0.191 & & & \\
\hline Yes & 75.7 & \pm 22.6 & & 27.2 & \pm 20.6 & & 20.5 & \pm 17.5 & & 43.9 & \pm 22.3 & & 25.3 & \pm 21.1 & 0.625 \\
\hline No & 73.3 & \pm 18.4 & & 32.3 & \pm 22.6 & & 19.8 & \pm 19.5 & & 49.1 & \pm 17.1 & & 26.9 & \pm 20.7 & \\
\hline Time since surgery less than 2 years & & & 0.174 & & & 0.901 & & & 0.873 & & & 0.476 & & & \\
\hline Yes & 72.7 & \pm 19.7 & & 28.2 & \pm 20.2 & & 20.6 & \pm 17.3 & & 45.1 & \pm 18.0 & & 27.4 & \pm 19.1 & 0.238 \\
\hline No & 76.6 & \pm 23.3 & & 28.2 & \pm 21.6 & & 20.6 & \pm 18.1 & & 44.6 & \pm 23.8 & & 24.7 & \pm 22.1 & \\
\hline
\end{tabular}

$\mathrm{M}=$ mean; $\mathrm{SD}=$ standard deviation; $\mathrm{p}=$ significance probability .

$S L B=$ sentinel lymph node biopsy; $\mathrm{PAD}=$ partial axillary dissection; $\mathrm{RAD}=$ radical axillary dissection; $\mathrm{BMI}=$ body mass index

EORTC QLC = European Organization for Research and Treatment of Cancer, Breast Cancer-Specific Quality of Life Questionnaire. 
The relation of the mean scores from the EORTC QLC 30 BR23 questionnaire scales with the subjective perceptions for the diagnosis of lymphedema, in relation to the time elapsed after surgical intervention, in women with breast cancer. Juiz de Fora, Minas Gerais, Brazil, 2013.

\begin{tabular}{|c|c|c|c|c|c|c|c|c|c|c|c|c|c|c|c|}
\hline \multirow{3}{*}{ Variables } & \multirow{2}{*}{\multicolumn{3}{|c|}{ Overall Health }} & \multicolumn{6}{|c|}{ EORTC 30} & \multicolumn{6}{|c|}{ BR23 } \\
\hline & & & & \multicolumn{3}{|c|}{ Functional } & \multicolumn{3}{|c|}{ Symptom } & \multicolumn{3}{|c|}{ Functional } & \multicolumn{3}{|c|}{ Symptom } \\
\hline & M & SD & $p$ & M & SD & $p$ & M & SD & $p$ & M & SD & $p$ & M & SD & $p$ \\
\hline Heavy feeling in the arm & & & $<0.001$ & & & $<0.001$ & & & $<0.001$ & & & $<0.001$ & & & $<0.001$ \\
\hline \multicolumn{16}{|l|}{ Currently } \\
\hline Yes & 60.60 & \pm 22.15 & & 42.90 & \pm 20.01 & & 33.50 & \pm 19.59 & & 54.70 & \pm 24.32 & & 44.00 & \pm 22.10 & \\
\hline No & 81.40 & \pm 19.10 & & 21.90 & \pm 18.20 & & 15.20 & \pm 13.84 & & 40.60 & \pm 19.38 & & 17.90 & \pm 14.96 & \\
\hline In the first 6 months after surgery & & & $<0.001$ & & & 0.002 & & & 0.004 & & & 0.158 & & & $<0.001$ \\
\hline Yes & 67.00 & \pm 23.91 & & 35.70 & \pm 23.03 & & 26.70 & \pm 20.51 & & 48.00 & \pm 22.05 & & 35.00 & \pm 24.10 & \\
\hline No & 81.70 & \pm 18.45 & & 22.60 & \pm 17.57 & & 16.00 & \pm 13.98 & & 42.60 & \pm 21.68 & & 18.70 & \pm 15.22 & \\
\hline Stretched skin sensation & & & $<0.001$ & & & $<0.001$ & & & $<0.001$ & & & 0.003 & & & $<0.001$ \\
\hline \multicolumn{16}{|l|}{ Currently } \\
\hline Yes & 65.20 & \pm 21.37 & & 41.40 & \pm 20.88 & & 31.40 & \pm 17.57 & & 54.20 & \pm 22.21 & & 40.40 & \pm 24.73 & \\
\hline No & 79.10 & \pm 21.26 & & 23.00 & \pm 18.79 & & 16.40 & \pm 16.09 & & 41.10 & \pm 20.69 & & 19.90 & \pm 16.27 & \\
\hline In the first 6 months after surgery & & & 0.046 & & & 0.002 & & & 0.029 & & & 0.146 & & & 0.022 \\
\hline Yes & 71.30 & \pm 21.90 & & 34.00 & \pm 21.76 & & 24.20 & \pm 18.46 & & 47.30 & \pm 22.53 & & 30.20 & \pm 22.69 & \\
\hline No & 78.90 & \pm 21.85 & & 22.60 & \pm 18.84 & & 17.20 & \pm 16.57 & & 42.50 & \pm 21.10 & & 21.40 & \pm 18.54 & \\
\hline Reduced hand / elbow movements & & & 0.002 & & & $<0.001$ & & & $<0.001$ & & & $<0.001$ & & & $<0.001$ \\
\hline \multicolumn{16}{|l|}{ Currently } \\
\hline Yes & 65.80 & \pm 23.71 & & 43.80 & \pm 20.60 & & 31.00 & \pm 18.61 & & 56.00 & \pm 24.27 & & 45.10 & \pm 22.71 & \\
\hline No & 79.70 & \pm 19.95 & & 20.70 & \pm 16.75 & & 15.70 & \pm 15.15 & & 39.50 & \pm 18.47 & & 16.40 & \pm 12.11 & \\
\hline In the first 6 months after surgery & & & 0.002 & & & $<0.001$ & & & $<0.001$ & & & $<0.001$ & & & $<0.001$ \\
\hline Yes & 69.00 & \pm 24.54 & & 35.60 & \pm 22.14 & & 26.10 & \pm 19.38 & & 51.40 & \pm 22.64 & & 33.70 & \pm 23.59 & \\
\hline No & 82.40 & \pm 16.34 & & 19.60 & \pm 15.91 & & 14.30 & \pm 13.30 & & 37.10 & \pm 18.28 & & 16.30 & \pm 12.39 & \\
\hline
\end{tabular}

$\mathrm{M}=$ mean; $\mathrm{SD}=$ standard deviation; $\mathrm{p}=$ significance probability

EORTC QLC = European Organization for Research and Treatment of Cancer, Breast Cancer-Specific Quality of Life Questionnaire. 
The relation of the mean scores from the EORTC QLC 30 BR23 questionnaire scales with the subjective perceptions for the diagnosis of lymphedema, in relation to the time elapsed after surgical intervention, in women with breast cancer. Juiz de Fora, Minas Gerais, Brazil, 2013.

\begin{tabular}{|c|c|c|c|c|c|c|c|c|c|c|c|c|c|c|c|}
\hline \multirow{3}{*}{ Variables } & \multirow{2}{*}{\multicolumn{3}{|c|}{ Overall Health }} & \multicolumn{6}{|c|}{ EORTC 30} & \multicolumn{6}{|c|}{ BR23 } \\
\hline & & & & \multicolumn{3}{|c|}{ Functional } & \multicolumn{3}{|c|}{ Symptom } & \multicolumn{3}{|c|}{ Functional } & \multicolumn{3}{|c|}{ Symptom } \\
\hline & M & SD & $p$ & M & SD & $p$ & M & SD & $p$ & M & SD & $p$ & M & SD & $p$ \\
\hline Tight shirt-sleeve sensation & & & $<0.001$ & & & $<0.001$ & & & $<0.001$ & & & $<0.001$ & & & $<0.001$ \\
\hline \multicolumn{16}{|l|}{ Currently } \\
\hline Yes & 58.70 & \pm 23.11 & & 46.90 & \pm 21.95 & & 39.10 & \pm 18.34 & & 59.80 & \pm 23.53 & & 48.80 & \pm 24.87 & \\
\hline No & 79.30 & \pm 19.95 & & 23.50 & \pm 18.09 & & 16.10 & \pm 14.45 & & 41.10 & \pm 19.85 & & 19.90 & \pm 15.46 & \\
\hline In the first 6 months after surgery & & & $<0.001$ & & & $<0.001$ & & & $<0.001$ & & & 0.021 & & & $<0.001$ \\
\hline Yes & 64.80 & \pm 24.41 & & 37.60 & \pm 21.61 & & 28.70 & \pm 19.40 & & 51.20 & \pm 24.06 & & 37.10 & \pm 23.68 & \\
\hline No & 82.50 & \pm 17.03 & & 21.50 & \pm 17.93 & & 15.00 & \pm 14.13 & & 40.30 & \pm 19.04 & & 17.60 & \pm 14.43 & \\
\hline $\begin{array}{l}\text { Tight sensation of ring / watch / } \\
\text { bracelet }\end{array}$ & & & $<0.001$ & & & $<0.001$ & & & $<0.001$ & & & 0.008 & & & $<0.001$ \\
\hline \multicolumn{16}{|l|}{ Currently } \\
\hline Yes & 59.80 & \pm 18.83 & & 43.60 & \pm 21.18 & & 35.70 & \pm 18.42 & & 56.90 & \pm 22.94 & & 47.40 & \pm 22.04 & \\
\hline No & 78.60 & \pm 21.40 & & 24.70 & \pm 19.48 & & 17.30 & \pm 15.90 & & 42.10 & \pm 20.78 & & 20.80 & \pm 17.56 & \\
\hline In the first 6 months after surgery & & & $<0.001$ & & & $<0.001$ & & & $<0.001$ & & & $<0.001$ & & & $<0.001$ \\
\hline Yes & 65.70 & \pm 23.23 & & 37.40 & \pm 20.64 & & 29.70 & \pm 17.96 & & 53.80 & \pm 22.50 & & 38.40 & \pm 21.83 & \\
\hline No & 80.40 & \pm 19.73 & & 23.10 & \pm 19.55 & & 15.60 & \pm 15.69 & & 39.90 & \pm 19.95 & & 18.70 & \pm 17.05 & \\
\hline
\end{tabular}

$M=$ mean; $S D=$ standard deviation; $p=$ significance probability.
EORTC QLC = European Organization for Research and Treatment of Cancer, Breast Cancer-Specific Quality of Life Questionnaire. 
Table 5

Results of the Simple Linear Regression model for the EORTC QLC 30 BR 23 in women treated for breast cancer. Juiz de Fora, Minas Gerais, Brazil, 2013.

\begin{tabular}{|c|c|c|c|c|c|}
\hline \multirow[b]{2}{*}{ Variables } & \multirow{2}{*}{$\begin{array}{l}\text { Overall Health } \\
\text { ( } p \text { value })\end{array}$} & \multicolumn{2}{|c|}{ EORTC 30} & \multicolumn{2}{|c|}{ BR23 } \\
\hline & & Functional & Symptoms & Functional & Symptoms \\
\hline \multirow[t]{2}{*}{ BMI } & NA & 2.356 & 1.678 & NA & 4.953 \\
\hline & & $(0.451)$ & $(0.552)$ & & $(0.014)$ \\
\hline \multirow[t]{2}{*}{ Without breast reconstruction } & NA & 7.432 & 8.167 & 10.998 & NA \\
\hline & & $(0.09)$ & $(0.12)$ & $(0.051)$ & \\
\hline \multirow[t]{2}{*}{ Heavy feeling in the arm, currently } & -16.394 & -10.233 & -9.772 & -7.893 & -11.252 \\
\hline & $(0.017)$ & $(0.082)$ & $(0.029)$ & $(0.121)$ & $(0.032)$ \\
\hline \multirow[t]{2}{*}{ Reduced hand / elbow movements, currently } & -11.987 & -18.622 & -9.419 & -12.290 & -20.468 \\
\hline & $(0.141)$ & $(<0.001)$ & $(0.007)$ & $(0.014)$ & $(<0.001)$ \\
\hline \multirow[t]{2}{*}{ Tight shirt-sleeve sensation, currently } & -12.896 & -7.345 & -15.639 & -5.456 & -11.737 \\
\hline & $(0.064)$ & $(0.093)$ & $(0.003)$ & $(0.134)$ & $(0.034)$ \\
\hline
\end{tabular}

$\mathrm{BMI}=$ body mass index; $\mathrm{NA}=$ not applicable.

EORTC QLC = European Organization for Research and Treatment of Cancer, Breast Cancer-Specific Quality of Life Questionnaire. 
lymphedema in the quality of life of this population.

Table 5 shows the variables associated with the poorer quality of life outcome, which remained in the final model after controlling for confounding factors (functional hand and time elapsed since surgery), according to the instrument's scales: high BMI (BR23 symptoms, $p=0.014$ ), no breast reconstruction (BR23 functional, $p=0.51$ ), heavy feeling in the arm (overall health, EORTC 30 and BR23 symptoms, with $p$ values of $0.017,0.029$, and 0.032 respectively), hand and elbow range of motion reduction (functional and symptoms in both modules, with $p$ values of $<0.001$ and $0.014 ; 0.007$ and $<0.001$, respectively), and a tight shirt-sleeve sensation at the arm (EORTC 30 and BR23 symptoms, with $p$ values of 0.003 and 0.034 ).

\section{Discussion}

Women over 50 years of age, white, with no stable marital relationship, with a low educational level, and homemakers or retirees were the main sociodemographic characteristics observed in the study population. Similar characteristics were observed by other authors in the literature. ${ }^{9-10}$

According to clinical characteristic, the majority of the population had a high BMI with a mean of $27.3 \mathrm{~kg} / \mathrm{m}^{2}$. Epidemiological studies associated excess weight and/or obesity, sedentary lifestyle, and poor eating habits with the increased incidence of cancer ${ }^{2}$ and the worst quality of life post-treatment. 11

The findings regarding tumor characteristics show that most of the women were diagnosed in advanced stages, III and IV. Users of the public health service have a higher prevalence of advanced stages, reflecting the longer time between suspected and confirmed diagnosis, and less frequent mammography when compared to the private sector. Consequently, this result in more invasive therapy 6,12 and reaffirms the deficiencies in integrated actions for cancer control in developing countries with respect to early detection, effective screening programs, and timely access to treatment. $2-3,13-14$

Most of the women underwent axillary lymphadenectomy and adjuvant therapy. The majority underwent conservative surgery, in general not reflecting the surgical approach most commonly observed in national studies, ${ }^{9-10}$ which can be explained by the quality of the information contained in the medical records and by the methodology used in the study, which considered only the first surgical procedure reported in the files.
In this study, health-related quality of life was assessed as generally good according to the instrument's scales, with the exception of the functional scale. Other studies corroborated with these findings. 7,9

Breast cancer patients are at high risk of developing changes in their physical and psychological functions, which negatively affect their overall quality of life. However, the literature indicates that overall quality of life tends to improve gradually over time. ${ }^{15}$ In the present study the mean elapsed time after surgery was 2.84 years, and according to Reid-Arndt et al., 16 after three years of treatment the overall quality of life is similar to the general population. The findings of the present study are in agreement with the literature, indicating high scores for the quality of life and overall health of women with breast cancer. 7,9

In a general way, adjuvant treatment is related to a reduced quality of life, 9,17 but the negative effects resulting from systemic treatment are mainly perceived during treatment due to the appearance of the symptoms such as pain, fatigue, nausea, vomiting, and changes in the emotional function, 9,18 and tend to progressively improve with time, after the treatment ends. 17 Therefore, the low symptom score, covered by the EORTC 30 dimension, by the patients, was expected since they were no longer under treatment, despite the high frequency of adjuvant therapy done by these women in our study. In addition, the low prevalence of lymphedema and self-reported symptoms of edema and arm pain may explain the low symptom score from the BR23 module.

It was also observed in the univariate analysis that only radiotherapy and the extent of axillary dissection were negatively associated with the quality of life via the symptoms dimension of the instrument, as observed in other studies. ${ }^{17}$

In this study, a negative assessment was shown from both the QLC30 functional scale, which focuses on physical, emotional, cognitive, and social aspects, and the BR23 functional scale, which addresses body image, sexual function, and future perspective.

Deficits in emotional, cognitive, and social function may be persistent to long-term in women with breast cancer, ${ }^{19}$ when compared to healthy women. ${ }^{20}$ In contrast, another study points to better scores via the SF-36 instrument for the components of mental and physical health for cancer survivors, compared to the general population, which can be explained by the greater appreciation of life upon going through cancer, as well as by the family support and the care 
received from the health professionals. 12

Many studies point to the negative effect on sexuality, resulting from the treatment, in the quality of life perceived by women with breast cancer. ${ }^{5,19,21-}$ 22 Problems of lack of sexual interest, vaginal lubrication, and pain at penetration are commonly reported after chemotherapy treatment 17,21 and tend to worsen over time, with the worst deficits perceived by younger women. $19-20$

Alopecia, breast deformities, and the loss of body symmetry are commonly reported by women with breast cancer, which imply a negative association of body image in QoL.5,21-22 Many studies affirm that body image is positively related to sexual satisfaction, ${ }^{19}$ to the possibility of breast reconstruction, $24-25$ and negatively related to depression. 17

The breast has a cultural and social meaning in the experience of female sexuality and any threat to its integrity is experienced with suffering, causing feelings of inferiority, rejection, and the loss of selfesteem. 19,23 Surgical mutilation and the secondary effects of systemic treatments result in major psycho-emotional impact in women with breas cancer, with respect to (re)elaboration of the body image and sexuality. 9 Although breast reconstruction is beneficial for quality of life, its practice is not a reality in the public health system, given the low frequency observed in this study and confirmed in others.6,9 Since 2013, there has been a legal requirement (Law 12.802) for breast reconstructive plastic surgery in cases of mutilation resulting from cancer.

Total mastectomy is associated with the worst QoL, when compared to conservative treatment, 25 which can be attributed to reduced sexual function 19 and more body image problems. ${ }^{24}$ Breast reconstruction, when compared to mastectomy without reconstruction, has better quality of life levels, ${ }^{26}$ with less impact on psychological aspects, on sexuality, attractiveness, and body image. 25

Although the analysis of this research cannot directly associate the scores from the quality of life subscale with breast reconstruction, another study, nevertheless, associated sexuality and body image as a dimension for the positive assessment of health and quality of life via the BR23 instrument. ${ }^{23}$ It can therefore be inferred that the less mutilating surgeries, the more quality of life the woman will enjoy, which was also observed by other authors. $9,19,25$ Another study, in contrast, affirms that there is no difference in satisfaction with the surgery and quality of life among the group of women who underwent conservative and reconstructive surgery, evaluated by the SF-36.26

A study evaluating QoL as a function of time after diagnosis, via the EORTC 30 BR23, demonstrated a decline in the emotional, social, and sexual domains of the functional scale, not only in the period of one to two years after initial treatment, but also after five years. 27 The functional scale of EORTC 30 BR23 was the only QoL domain that did not show improvement over time after the initial diagnosis, with the worst scores being perceived in body image, sexual function, and future perspective. This fact is corroborated by a study carried out in 2012,22 in which it was highlighted that body image has implications for the assessment of health and quality of life, being a relationship in the opposite direction, while sexual function and pleasure seem to be positively related according to the EORTC 30 . Another study, however, found high scores for the functional scale, which may be explained by the mean time elapsed after diagnosis ( 4.8 years) and by the sample selection, which took place in a public institution with extensive professional support in the pre- and post-operative period for this population.7

Regarding clinical variables, high BMI was broadly associated with quality of life. Obesity is indicated as a determinant of risk for breast cancer, ${ }^{2}$ and the presence of physical and metabolic comorbidities. 27 Studies show positive associations with weight, more often seen in postmenopausal women. 1,3-4,7,28 Excess weight and lack of physical activity in this population adversely affect quality of life and predispose it to worse prognoses for cancer. In a retrospective study, the prevalence of weight gain increases over time in women with breast cancer, after the initial treatment. 28 The side effects of treatment adversely affect physical function, body weight, and cardiovascular health. 28 This impairment tends to negatively affect the performance of activities of daily life and work, in addition to compromising healthy life behaviors, such as regular physical exercise, in these patients. ${ }^{29}$

With respect to lymphedema, its prevalence after treatment in this study was $7.4 \%$, lower than that reported in a previous study carried out at the same health institution, of $44.8 \% .10$ Although some authors affirm that size measurement and estimated limb volume are equally valid instruments with excellent reproducibility, 28 the difference in the choice of the diagnostic method used to characterize lymphedema between studies may explain the divergence of the values found. Another factor that may also explain this difference in prevalence concerns the follow-up time of the study, since the frequency of lymphedema tends to increase with the time from the initial lesion of the axillary lymph nodes via surgery or radiotherapy.10,29 Thus, the longer the 
follow-up period, the greater the probability of cases of lymphedema, as verified in the study by Paiva et al.10 However, the information from the present study is in agreement with other sectional studies, which report a frequency between $0 \%$ and $22 \%$ of the cases. ${ }^{28}$

Disagreement between subjective complaints of lymphedema and the findings upon physical examination can occur. Although the data from the literature may be conflicting, the importance of both methods is not excluded, as subjective patient complaints may precede the ability to detect objective changes from lymphedema and minimize its progression. 28

Arm pain, sensation of heaviness, restricted shoulder amplitude, and edema are symptoms frequently reported by women with breast cancer and are commonly associated with the presence of lymphedema, corroborating results similarly found in the present study.5,30

Considering that health-related quality of life, according to the World Health Organization (WHO), is a multidimensional construct, of a highly subjective nature and changeable over time, the subjective perceptions of changes in the upper limb, in this study, were predictive of negative impact on the of overall health, physical function (EORTC30 and 23), and functional (EORTC30 and 23) dimensions of HRQoL.

Research conducted by Assis et al.30 yielded results similar to this study, in which complaints of pain, weakness, numbness, and shoulder range of motion (ROM) reduction were correlated with overall health, physical, and functional (EORTC QLQ-C30 ) dimensions, and body image, upper limb symptoms, and future perspective (BR23).

After controlling for possible confounding, breast reconstruction, body mass index (BMI), tight shirt-sleeve sensation, heavy feeling in the arm, and shoulder ROM reduction were able to explain HRQoL, as observed in other studies. 6,9-10,12,15-17,19-20

Although HRQoL is relatively good at six months after surgical intervention, subjective perceptions have proven to be predictors of greater negative influence for women with breast neoplasia, in addition to factors such as BMI and breast reconstruction.

Another interesting aspect of this study lies in the implications its results may have for the development of therapies promoting better adaptation to the disease and to cancer treatment. Thus, such knowledge can contribute to the production of integral care for breast cancer patients through health team follow-up protocols in the pre- and post-operative period, in order to prevent, reduce, or minimize functional alterations, social disability, and to promote the psychosocial well-being of these women.

It should be noted that the absence of a cut-off point for the study population in the EORTC QLC 30 BR23 instruments used makes it difficult to establish more precise assessments, and limits comparability and extrapolation of the results. The absence of a prior evaluation of the BMI and the measurement of the volume of the affected arm is another limitation of the study. In order to meet these demands, further studies must be carried out.

\section{References}

1. Instituto Nacional de Câncer José Alencar Gomes da Silva. Estimativa 2016: incidência de câncer no Brasil. Rio de Janeiro: Inca; 2015.

2. Tiezzi DG. Câncer de mama: um futuro desafio para o sistema de saúde nos países em desenvolvimento. Rev Bras Ginecol Obstet. 2010; 32 (6): 257-9.

3. Oliveira EXG, Pinheiro RS, Melo ECP, Carvalho MS Condicionantes socioeconômicos e geográficos do acesso à mamografia no Brasil, 2003-2008. Ciênc Saúde Coletiva. 2011; 16 (9): 3649-64.

4. Bertan FC, Castro EK. Qualidade de vida e câncer: revisão sistemática de artigos brasileiros. Psico. 2009; 40 (3): 366 72.

5. Vieira RAC, Silva FCB, Biller G, Silva JJ, Paiva CE, Sarri AJ. Instrumentos de avaliação quantitativa e qualitativa das sequelas relacionadas ao tratamento do câncer de mama.

Rev Bras Mastol. 2016; 26 (3): 126-32.

6. Sousa E, Carvalho FN, Bergmann A, Fabro EAN, Dias RA, Koifman RJ. Funcionalidade de membro superior em mulheres submetidas ao tratamento do câncer de mama. Rev Bras Cancerol. 2013; 59 (3): 409-17.

7. Michels FAS, Latorre MRDO, Maciel MS. Validity, reliability and understanding of the EORTC-C30 and EORTCBR23, quality of life questionnaires specific for breast cancer. Rev Bras Epidemiol. 2013; 16 (2): 352-63.

8. Rockson SG, Miller LT, Senie R, Brennan MJ, CasleySmith JR, Földi E, Földi M, Gamble GL, Kasseroller RG, Leduc A, Lerner R, Mortimer PS, Norman SA, Plotkin CL, Rinehart-Ayres ME, Walder AL. American Cancer Society Lymphedema Workshop. Workgroup III: diagnosis and management of lymphedema. Cancer. 1998; 83 (12 Supp1 American): 2882-5. 
9. Bezerra KB, Silva DSM, Chein MBC, Ferreira PR, Maranhão JKP, Ribeiro NL, Mochel EG. Qualidade de vida de mulheres tratadas de câncer de mama em uma cidade do nordeste do Brasil. Ciênc Saúde Coletiva. 2013; 18 (7): 1933-41.

10. Paiva DMF, Leite ICG, Rodrigues VO, Cesca MG. Fatores associados ao linfedema em pacientes com câncer de mama. Rev Bras Ginecol Obstet. 2011; 33 (2): 75-80.

11. Connor AE, Baumgartner RN, Pinkston CM, Boone SD, Baumgartner KB. Obesity, ethnicity, and quality of life among breast cancer survivors and women without breast cancer: the long-term quality of life follow-up study. Cancer Causes Control. 2016; 27 (1): 115-24.

12. Soares PBM. Avaliação clínico-epidemiológica e qualidade de vida em mulheres com câncer de mama. Rev Bras Ginecol Obstet. 2011; 33 (3).

13. Guerra MR, Silva GA, Nogueira MC, Leite ICG, Oliveira RVC, Cintra JRD, Teixeira MTB. Sobrevida por câncer de mama e iniquidade em saúde. Cad Saúde Pública. 2015; 31 (8): 1673-84.

14. Gonzaga CMR, Junior RF, Curado MP, Sousa ALL, Neto JAS, Souza MR. Temporal trends in female breast cancer mortality in Brazil and correlations with social inequalities: ecological time-series study. BMC Public Health. 2015; 15: 96.

15. Chopra I, Kamal KM. A systematic review of quality of life instruments in long-term breast cancer survivors. Health Qual Life Outcomes. 2012; 10: 14.

16. Reid-Arndt SA, Hsieh C, Perry MC. Neuropsychological functioning and quality of life during the first year after completing chemotherapy for breast cancer. Psychooncology. 2010; 19 (5): 535-44.

17. Lotti RCB, Barra AA, Dias RC, Makluf ASD. Impacto do tratamento de câncer de mama na qualidade de vida. Rev Bras Cancerol. 2008; 54 (4): 367-71.

18. Lôbo AS, Fernandes AF, Almeida PC, Carvalho CM, Sawada NO. Qualidade de vida em mulheres com neoplasias de mama em quimioterapia. Acta Paul Enferm. 2014; 27 (6): 554-9.

19. Cesnik VM, Santos MA. Mastectomia e sexualidade: uma revisão integrativa. Psicol Reflex Crit. 2012; 25 (2): 33949

20. Kluthcovsky ACGC, Urbanet, AAL. Qualidade de vida em pacientes sobreviventes de câncer de mama comparada à de mulheres saudáveis. Rev Bras Ginecol Obstet. 2012; 34 (10): 453-8

Received on November 10, 2016

Final version submitted on March 6, 2017

Approved on March 27, 2017
21. Winch CJ, Sherman KA, Koelmeyer LA, Smith KM, Mackie H, Boyages J. Sexual concerns of women diagnosed with breast cancer-related lymphedema. Support Care Cancer. 2015; 23: 3481-91

22. Garcia SN, Jacowski M, Castro GC, Galdino C, Guimarães PRB, Kalinke LP. Os domínios afetados na qualidade de vida de mulheres com neoplasia mamária. Rev Gaúcha Enferm. 2015; 36 (2): 89-96.

23. Remondes-Costa S, Jimenez F, Pais-Ribeiro JL. Imagem corporal, sexualidade e qualidade de vida no cancro da mama. Psicol Saúde Doenças. 2012; 13 (2): 327-39.

24. Fang SY, Shu BC, Chang YJ. The effect of breast reconstruction surgery on body image among women after mastectomy: a meta-analysis. Breast Cancer Res Treat. 2013; 137 (1): 13-21.

25. Simeão SFAP, Landro ICR, De Conti MHS, Gatti MAN, Delgallo WD, de Vitta A. Qualidade de vida em grupos de mulheres acometidas de câncer de mama. Ciênc Saúde Coletiva. 2013; 18 (3): 779-88.

26. Freitas-Silva R. Qualidade de vida, satisfação com a cirurgia e morbidade no ombro e braço de mulheres com câncer de mama submetidas à quadrantectomia ou à mastectomia com reconstrução imediata. Rev Bras Ginecol Obstet. 2010; 32 (2): 99.

27. Schmitz KH, Speck RM, Rye SA, DiSipio T, Hayes SC. Prevalence of breast cancer treatment sequelae over 6 years of follow-up: the Pulling Through Study. Cancer. 2012; 118 (8 Suppl): 2217-25.

28. Bergmann A, Mattos IEM, Koifman RJ. Diagnóstico do linfedema: análise dos métodos empregados na avaliação do membro superior após linfadenectomia axilar para tratamento do câncer de mama. Rev Bras Cancerol. 2004; 50 (4): 311-20.

29. Hayes SC, Johansson K, Stout NL, Prosnitz R, Armer JM, Gabram S, Schmitz KH. Upper-body morbidity after breast cancer: incidence and evidence for evaluation, prevention, and management within a prospective surveillance model of care. Cancer. 2012; 118 (8 Suppl): 2237-49.

30. Assis MR, Marx AG, Magna LA, Ferrigno IS. Late morbidity in upper limb function and quality of life in women after breast cancer surgery. Braz J Phys Ther. 2013; 17 (3): 236-43. 